\title{
Perfil das exposições a medicamentos por mulheres em idade reprodutiva atendidas por um Centro de Informações Toxicológicas
}

\author{
Profile of exposure to medication among women \\ of reproductive age attended in a Toxicologial Information Center
}

Carina Harumi Takahama ${ }^{1}$

Conceição Aparecida Turini ${ }^{1}$

Edmarlon Girotto ${ }^{2}$

\footnotetext{
${ }^{1}$ Departamento de Patologia Aplicada, Análise Clínicas e Toxicológicas, Centro de Ciências da Saúde, Universidade Estadual de Londrina. Av. Robert Koch 60, Vila Operária. 86.038-350 Londrina PR Brasil. carina.cht@gmail.com ${ }^{2}$ Departamento de Ciências Farmacêuticas, Centro de Ciências da Saúde, Universidade Estadual de Londrina.
}

\begin{abstract}
The scope of this article is to analyze the epidemiological data relating to exposure to medication among women of reproductive age attended at a Toxicology Information Center. A study was conducted among women of reproductive age exposed to medication between 2007 and 2011. The variables relating to the patients, the occurrence and the medication involved were studied in a total of 777 notified cases. Data was collected from Aggravated Injury Notification System forms and processed on Epi Info for Windows software. The majority of the occurrences (90.5\%) was intentional, 33.7\% of theses incidents involved the intake of 2 or 3 types of drugs by the patients and the percentile of hospitalization was $35.6 \%$. Drugs acting on the central nervous system were responsible for $59.9 \%$ of the incidents, and antidepressants (21.3\%) and antiepileptics (21.2\%) were most commonly involved. The main factors associated with hospitalization were: delayed medical rescue after exposure, patients with higher education, ingestion of 2 or 3 types of drugs and exposure to anti-epileptics and antidepressants. Data from this study showed that exposure to medication is a serious health problem for women of reproductive age and it contributes to the increase in the number of hospitalizations.
\end{abstract}

Key words Women, Reproductive age, Intoxication, Hospitalization, Information systems
Resumo O objetivo deste artigo é descrever os dados epidemiológicos referentes às exposições a medicamentos por mulheres em idade fértil atendidas por um Centro de Informações Toxicológicas. Realizou-se um estudo com mulheres em idade fértil, expostas a medicamentos entre 2007 e 2011. Foram estudadas variáveis relacionadas às pacientes, à ocorrência e ao medicamento envolvido, totalizando 777 notificações. Os dados foram obtidos do Sistema de Informação de Agravos de Notificação e processadas com o uso do programa Epi Info. A maioria das ocorrências (90,5\%) foi intencional, em 33,7\% dos casos foram utilizados de dois a três medicamentos e a frequência de hospitalização foi de 35,6\%. Os medicamentos com atuação no Sistema Nervoso Central foram responsáveis por 59,9\% das ocorrências, destacando-se os antiepilépticos (21,2\%) e os antidepressivos (20,7\%). Os principais fatores associados à hospitalização das pacientes foram: demora no atendimento após a ocorrência da exposição, pacientes com nível superior, contato com dois ou mais medicamentos e exposições a antiepilépticos $e$ antidepressivos. Os resultados obtidos mostraram que as exposições a medicamentos representam um grave problema à saúde de mulheres em idade fértil e contribuem para o aumento das internações hospitalares.

Palavras-chave Mulheres, Idade fértil, Intoxicação, Hospitalização, Sistemas de Informação 


\section{Introdução}

Os medicamentos exercem um importante papel nos sistemas sanitários por constituírem a ferramenta terapêutica mais empregada para a manutenção e a recuperação das condições de saúde, sendo responsáveis por parte significativa da melhora da qualidade e expectativa de vida da população ${ }^{1-5}$.

Entretanto, seu uso irracional, indevido ou abusivo pode torná-los potentes causadores de danos à saúde e de óbitos ${ }^{3,6-8}$. Segundo a Organização Mundial da Saúde $(\mathrm{OMS})^{9}$, os hospitais gastam de 10 a $40 \%$ de seus orçamentos em complicações causadas pelo mau uso de medicamentos.

No Brasil, merecem destaque a facilidade de aquisição de medicamentos sob prescrição médica, a propaganda de medicamentos, o padrão do consumo de medicamentos pela população, caracterizado pela automedicação, polifarmácia e o uso indevido e indiscriminado de determinados fármacos como antibióticos e psicotrópi$\cos ^{1,3,8,10}$. Estes aspectos contribuem para que os medicamentos sejam os principais agentes responsáveis por intoxicações, com consequente aumento do número de atendimentos nas unidades de urgência e emergência, hospitalizações e óbitos registrados em nosso país, ${ }^{6,811-13}$.

Segundo as estatísticas divulgadas pelo Sistema Nacional de Informações Toxico-Farmacológicas (Sinitox), os medicamentos ocupam, desde 1994, o primeiro lugar no conjunto dos agentes responsáveis por intoxicações, respondendo por, aproximadamente, $27 \%$ dos casos registrados no Brasil ${ }^{14-16}$.

Entre 2007 e 2011 os medicamentos foram os principais agentes desencadeadores em $37,4 \%$ dos casos de intoxicação notificados (209.356 casos) e responsáveis pela segunda causa de óbito por intoxicação $(23,9 \%)^{17}$. Dentre estes casos, $62,9 \%$ eram pacientes do sexo feminino, com a grande maioria $(96,0 \%)$ pertencente à faixa etária reprodutiva ${ }^{17}$.

Os medicamentos preocupam também, há algum tempo, as autoridades e os profissionais de saúde de outros países, pelo aumento do volume dos casos de intoxicação e óbitos que este agente provoca, colocando-os em destaque nas estatísticas mundiais ${ }^{7,18-21}$. Na maioria dos países, e em especial no Brasil, as estatísticas divulgadas pelos sistemas oficiais de notificação não refletem a real magnitude do problema, devido à subnotificação e à tendência de registro apenas dos casos mais agudos, com sinais clínicos mais exuberantes. A falta de estratégias para o contro- le e prevenção das intoxicações tem levado a um número crescente e negligenciado dessas ocorrências, tanto pela sociedade quanto pelas autoridades competentes ${ }^{21,22}$.

No Brasil, as mulheres em idade reprodutiva (14 a 49 anos) representam $65 \%$ do total da população feminina e constituem um segmento social importante para a elaboração das políticas de saúde ${ }^{23}$. Como os medicamentos são os principais agentes determinantes de intoxicações agudas e, em mulheres, uma das causas mais frequentes de lesões e intoxicações autoinfligidas, tornam-se importantes estudos que avaliem as intoxicações neste grupo populacional. Desta forma, esta investigação objetivou descrever os dados epidemiológicos referentes à exposição de mulheres em idade fértil a medicamentos que foram atendidas por um Centro de Informações Toxicológicas.

\section{Metodologia}

Realizou-se um estudo retrospectivo, descritivo e exploratório com dados secundários obtidos no Sistema de Informação de Agravos de Notificação (SINAN). Avaliaram-se os registros de pacientes do sexo feminino, em idade reprodutiva, e expostas a medicamentos e atendidas direta ou indiretamente pelo Centro de Informações Toxicológicas de Londrina (CIT-Londrina), Paraná.

Os Centros de Informação e Assistência Toxicológica (CIATs) são unidades públicas de referência em Toxicologia Clínica, de abrangência estadual ou regional, com atendimento em regime de plantão permanente, por meio telefônico e/ou presencial, das intoxicações e envenenamentos. O CIT-Londrina atende 81 municípios pertencentes a quatro Regionais de Saúde que compõem a macrorregião norte do estado do Paraná, e abrange uma população estimada de 1.723.029 habitantes.

Os casos atendidos e registrados no CIT-Londrina no período de janeiro de 2007 a dezembro de 2011 foram analisados. O período inicial de análise deve-se ao fato de em janeiro de 2007 as notificações deste CIT passarem a ser realizadas nas fichas de notificação do SINAN para Intoxicações.

No período de estudo o CIT-Londrina registrou 7.682 atendimentos decorrentes da exposição a diversos agentes tóxicos e circunstâncias, com 24,5\% (1882) de casos relacionados a medicamentos. Destes, as mulheres em idade fértil representaram $41,3 \%$ dos pacientes expostos, to- 
talizando 778 atendimentos. Um caso foi excluído por apresentar dados inconsistentes, gerando um total de 777 casos.

As fichas utilizadas no estudo foram localizadas e analisadas individualmente, considerando-se as seguintes variáveis independentes: idade (10 a 14 anos, 15 a 19 anos, 20 a 29 anos, 30 a 39 anos, 40 a 49 anos), gestação (sim ou não), período gestacional $\left(1^{\circ}, 2^{\circ}\right.$ e $3^{\circ}$ trimestres $)$, grau de escolaridade (ensino fundamental, médio ou superior), intenção (intencional ou não intencional), circunstância, tempo decorrido entre a exposição e o atendimento (até uma hora, de duas a cinco horas, seis horas ou mais), evolução clínica, quantidade de medicamentos envolvidos, associação com outros agentes tóxicos, tipo de controle na aquisição do medicamento (isento de prescrição, sob prescrição médica ou venda com retenção de receita) e classe farmacológica.

A variável dependente (desfecho) analisada foi a ocorrência ou não de hospitalização decorrente da exposição e/ou intoxicação a medicamentos. Como sistema de classificação dos fármacos responsáveis pelas exposições adotou-se o terceiro nível da Anatomical Therapeutic Chemical (ATC) da Organização Mundial de Saúde ${ }^{24}$.

As informações coletadas foram transcritas em banco de dados criado no programa Epi Info para Windows versão 3.5.1. e processadas e analisadas com o uso do mesmo programa. As associações entre variáveis foram analisadas com o teste de qui-quadrado, sendo considerados significativos os testes que apresentaram valores de $\mathrm{p}<0,05$.

\section{Resultados}

Dos 777 casos analisados, a idade média das pacientes foi de 26,5 anos (Desvio-Padrão = 9,95), com uma maior frequência na faixa etária de 20 a 29 anos (33,6\%). Em 39\% dos casos, as pacientes possuíam escolaridade até o ensino médio, porém, observou-se falta de informações para esta categoria em grande parte das fichas de notificação (51,6\%).

As pacientes gestantes totalizaram $27(3,5 \%)$ dos casos, com $12(44,4 \%)$ no primeiro trimestre de gestação. Em 309 fichas analisadas $(39,8 \%)$ esta variável (gestação) foi notificada como ignorada (Tabela 1 ).

No detalhamento das informações relacionadas à exposição, também descritas na Tabela 1 , verificou-se que a maioria das ocorrências $(90,5 \%)$ foi intencional, principalmente pelas tentativas de suicídio (90,0\%). Identificou-se o uso dos medicamentos com outras substâncias químicas em $59(7,6 \%)$ ocorrências, sendo a maior parte com bebida alcoólica $(69,5 \%)$. A grande maioria $(78,0 \%)$ das pacientes recebeu atendimento médico em até cinco horas após a exposição.

Observou-se o uso de apenas um medicamento por $60,6 \%$ das pacientes, total de 1290 medicamentos (média $=1,66$ ), com o máximo de nove medicamentos. Quanto ao tipo de controle necessário aos medicamentos utilizados, de 1289 identificados, constatou-se um elevado percentual de uso de fármacos que requerem maior rigor na sua dispensação $(62,5 \%)$ (Figura 1).

A frequência de hospitalização foi de $35,6 \%$ $(\mathrm{n}=277)$, sendo que destas, $62,1 \%$ ficaram hospitalizadas dois dias ou mais. A classificação final dos casos mostrou que 79,9\% tiveram into-

Tabela 1. Distribuição dos casos de exposição por mulheres em idade fértil de acordo com as variáveis relacionadas às pacientes e à exposição,

CIT-Londrina, 2007-2011.

\begin{tabular}{|c|c|c|}
\hline \multirow{2}{*}{ Características } & \multicolumn{2}{|c|}{$\begin{array}{l}\text { Casos } \\
\text { registrados }\end{array}$} \\
\hline & $\mathbf{N}$ & $\%$ \\
\hline \multicolumn{3}{|l|}{ Faixa etária } \\
\hline 10 a 14 & 70 & 9,0 \\
\hline 15 a 19 & 168 & 21,6 \\
\hline 20 a 29 & 261 & 33,6 \\
\hline 30 a 39 & 168 & 21,6 \\
\hline 40 a 49 & 110 & 14,2 \\
\hline \multicolumn{3}{|l|}{ Escolaridade } \\
\hline Ensino fundamental & 127 & 16,3 \\
\hline Ensino médio & 176 & 22,7 \\
\hline Ensino superior & 73 & 9,4 \\
\hline Ignorado & 401 & 51,6 \\
\hline \multicolumn{3}{|l|}{ Gestante } \\
\hline $\operatorname{Sim}$ & 27 & 3,5 \\
\hline Não & 441 & 56,8 \\
\hline Ignorado & 309 & 39,8 \\
\hline \multicolumn{3}{|l|}{ Intenção } \\
\hline Intencional & 703 & 90,5 \\
\hline Não intencional & 58 & 7,5 \\
\hline Indeterminada & 16 & 2,1 \\
\hline \multicolumn{3}{|c|}{ Associação não medicamentosa } \\
\hline Sim & 59 & 7,6 \\
\hline Não & 717 & 92,7 \\
\hline \multicolumn{3}{|c|}{$\begin{array}{l}\text { Tempo decorrido entre a exposição } \\
\text { e o atendimento }\end{array}$} \\
\hline Até uma hora & 289 & 40,4 \\
\hline De duas a cinco horas & 269 & 37,6 \\
\hline Seis horas ou mais & 157 & 22,0 \\
\hline
\end{tabular}

* 62 pacientes com tempo de história indeterminado. 
xicação confirmada. Em relação à evolução clínica, a cura sem sequela representou 97,3\% dos casos, registrando-se apenas dois $(0,3 \%)$ óbitos (Tabela 2).

Os medicamentos com atuação no Sistema Nervoso Central (SNC) foram responsáveis por 59,9\% das ocorrências. Neste, destacaram-se os seguintes grupos farmacológicos: antiepilépticosN03A (21,2\%), antidepressivos-N06A (20,7\%), ansiolíticos-N05B (8,1\%), analgésicos e antipiréticos-N02B $(7,4 \%)$ e antipsicóticos-N05A $(7,2 \%)$. Também apresentaram importante incidência os anti-inflamatórios não-esteroidaisM01A (5,1\%). Os princípios ativos mais envolvidos nas exposições/intoxicações foram: clonazepan (10,5\%), amitriptilina $(7,8 \%)$, fluoxetina

Tipo de controle para dispensação* $(\mathrm{N}=777)$

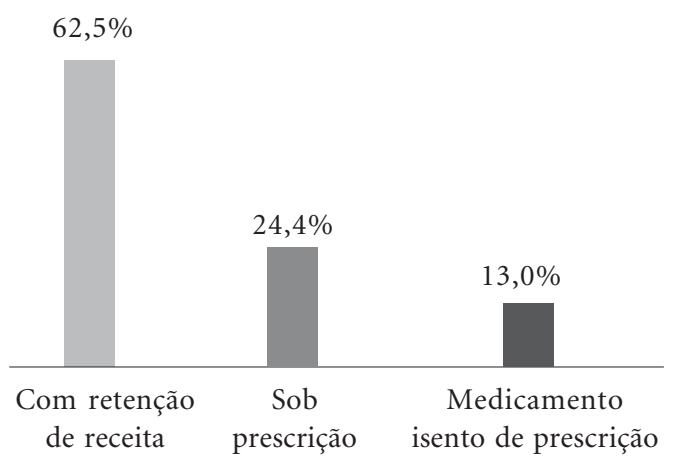

Número de medicamentos utilizados $(\mathrm{N}=1.289)$

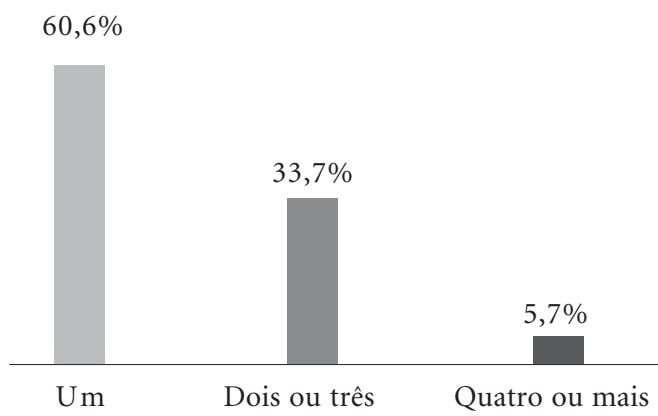

*Um medicamento não identificado.

Figura 1. Distribuição das ocorrências de exposição por mulheres em idade fértil de acordo com os medicamentos envolvidos, CIT-Londrina, 2007-2011.
$(5,7 \%)$, carbamazepina $(4,9 \%)$, diazepam $(4,2 \%)$, paracetamol (3,3\%), fenobarbital $(2,9 \%)$, dipirona $(2,1 \%)$ e haloperidol (2,0\%).

$\mathrm{Na}$ Tabela 3 apresentam-se os fatores associados à hospitalização. Nota-se que a demora no atendimento após ocorrência da intoxicação/exposição, pacientes com maior escolaridade e exposição a mais de um medicamento mostraram-se associados ao maior risco de hospitalização. Também se mostrou significativa a exposição a antidepressivos e antipilépticos.

\section{Discussão}

Considerando os dados registrados pelos sistemas oficiais de informação em saúde, apesar da elevada subnotificação, e os resultados apresentados em vários estudos, nacionais e internacionais, observa-se uma preocupação crescente com as intoxicações medicamentosas, tanto em relação à demanda dos serviços, como com o custo financeiro para o poder público. Esses dados evidenciam ainda a predominância deste tipo de intoxicação no gênero feminino, que representa também a maioria das hospitalizações por essas causas $^{13,25-29}$.

No presente estudo constatou-se uma importante participação de fármacos com atuação

Tabela 2. Evolução das ocorrências de exposição a medicamentos por mulheres em idade fértil, CIT-Londrina, 2007-2011.

\begin{tabular}{lrr}
\hline \multirow{2}{*}{ Características } & \multicolumn{2}{c}{ Casos } \\
& \multicolumn{1}{c}{ registrados } \\
\cline { 2 - 3 } & N & \% \\
\hline Hospitalização & & \\
Sim & 177 & 35,6 \\
Um dia & 125 & 37,9 \\
$\quad$ Dois a três dias & 47 & 17,0 \\
$\quad$ Quatro dias ou mais & 500 & 64,4 \\
Não & & \\
Classificação final & 621 & 79,9 \\
Intoxicação confirmada & 155 & 19,9 \\
Somente exposição & 1 & 0,1 \\
Reação adversa ao medicamento & & \\
Evolução do caso & 756 & 97,3 \\
Cura sem sequela & 2 & 0,3 \\
Obito por intoxicação & 15 & 1,9 \\
Perda de seguimento & 4 & 0,5 \\
Ignorado & & \\
\hline
\end{tabular}


Tabela 3. Frequência de hospitalização de acordo com as variáveis relacionadas às pacientes e aos medicamentos em mulheres em idade fértil, CIT-Londrina, 2007-2011.

\begin{tabular}{|c|c|c|c|}
\hline \multirow[t]{2}{*}{ Variáveis independentes } & \multicolumn{2}{|c|}{ Hospitalização (\%) } & \multirow{2}{*}{ Valor $\mathbf{p}$} \\
\hline & Sim & Não & \\
\hline \multicolumn{4}{|c|}{ Características relacionadas à exposição e paciente } \\
\hline \multicolumn{4}{|l|}{ Tempo de atendimento } \\
\hline Em até uma hora & 29,1 & 70,9 & $<0,05$ \\
\hline A partir de duas horas & 39,5 & 60,5 & \\
\hline \multicolumn{4}{|l|}{ Gestante } \\
\hline Sim & 44,4 & 55,6 & 0,440 \\
\hline Não & 35,1 & 64,9 & \\
\hline \multicolumn{4}{|l|}{ Ensino superior } \\
\hline Sim & 53,4 & 46,6 & $<0,05$ \\
\hline Não & 35,3 & 64,7 & \\
\hline \multicolumn{4}{|l|}{ Associação não medicamentosa } \\
\hline Sim & 37,3 & 62,7 & 0,884 \\
\hline Não & 35,4 & 64,6 & \\
\hline \multicolumn{4}{|l|}{ Associação com bebidas alcoólicas } \\
\hline $\operatorname{Sim}$ & 19 & 46,3 & 0,142 \\
\hline Não & 258 & 35,1 & \\
\hline \multicolumn{4}{|l|}{ Tentativa de suicídio } \\
\hline Sim & 36,8 & 63,2 & 0,069 \\
\hline Não & 25,6 & 74,4 & \\
\hline \multicolumn{4}{|c|}{ Características relacionadas aos medicamentos } \\
\hline \multicolumn{4}{|l|}{ Número de medicamentos } \\
\hline $\mathrm{Um}$ & 31,4 & 68,6 & $<0,05$ \\
\hline Dois ou mais & 42,2 & 57,8 & \\
\hline \multicolumn{4}{|l|}{ Antiepilépticos - N03A } \\
\hline Sim & 110 & 44,7 & $<0,001$ \\
\hline Não & 167 & 31,5 & \\
\hline \multicolumn{4}{|l|}{ Antidepressivos - N06A } \\
\hline Sim & 107 & 42,3 & $<0,05$ \\
\hline Não & 170 & 32,4 & \\
\hline \multicolumn{4}{|l|}{ Ansiolíticos - N05B } \\
\hline Sim & 39 & 38,2 & 0,559 \\
\hline Não & 238 & 35,3 & \\
\hline \multicolumn{4}{|l|}{ Analgésicos e antipiréticos - N02B } \\
\hline $\operatorname{Sim}$ & 30 & 37,5 & 0,715 \\
\hline Não & 247 & 35,4 & \\
\hline \multicolumn{4}{|l|}{ Antipsicóticos - N05A } \\
\hline Sim & 36 & 43,4 & 0,120 \\
\hline Não & 241 & 34,7 & \\
\hline \multicolumn{4}{|c|}{ Anti-inflamatórios não esteroidais - M01A } \\
\hline $\operatorname{Sim}$ & 15 & 25,4 & 0,088 \\
\hline Não & 262 & 36,5 & \\
\hline
\end{tabular}

no SNC, totalizando quase $60 \%$ das exposições. Vários estudos evidenciam que esses fármacos representam a parcela mais significativa no total geral das intoxicações medicamentosas no Brasil $^{13,26,27}$. Numa análise mais pormenorizada feita a este grupo, destacaram-se os antidepressi- vos, antiepiléticos e antipsicóticos. Em estudo de base populacional, McClure et al. ${ }^{28}$ evidenciaram que $22,9 \%, 19,9 \%$ e $17,4 \%$ das intoxicações em mulheres em idade reprodutiva envolveram analgésicos, tranquilizantes benzodiazepínicos e antidepressivos, respectivamente. 
O fato de pacientes depressivos, muitas vezes, tentarem suicídio aponta para que a prescrição de medicamentos, principalmente para esses casos, seja racionalizada, com quantidades adequadas para a sua correta utilização ${ }^{30}$. Ainda que vários estudos sustentem uma relação direta entre o abuso de substâncias psicoativas e comportamentos suicidas, principalmente entre jovens, a natureza desta relação não está claramente estabelecida, e requer mais investigações ${ }^{27,31}$.

O Brasil apresenta um elevado consumo de psicofármacos ${ }^{5,32} \mathrm{e}$, apesar da elevada frequência de ocorrências de intoxicações por este grupo, estas substâncias não são necessariamente mais tóxicas. Entretanto, o fato dos benzodiazepínicos e antidepressivos serem classes de produtos muito prescritos faz com que esta alta acessibilidade gere um maior risco para ingestões excessivas e sobredosagem. Do ponto de vista toxicológico, é importante ressaltar que nestas duas classes de fármacos existem várias substâncias que, na sua maioria, são extensamente metabolizadas no organismo, originando compostos farmacologicamente ativos que contribuem para o desenvolvimento de intoxicações.

Estudo realizado por Fernandes et al..$^{26}$ detectou o predomínio do uso de tranquilizantes e antidepressivos em relação a outros grupos de medicamentos nas intoxicações analisadas, com destaque para a ingestão de amitriptilina, o que concorda com a presente investigação. Este agente foi responsável pelo maior número de óbitos e dias de hospitalização ocorridos neste período, e verificou-se tratar-se de um fármaco de fácil acesso, baixo custo, e também distribuído gratuitamente pelo sistema público de saúde.

Pacientes com transtornos mentais representam um grupo altamente propenso às tentativas de suicídio ${ }^{33}$, sendo a depressão o distúrbio mais comum. Desta forma, é importante o frequente monitoramento deste paciente, incluindo o apoio familiar, de cuidadores e de equipes de saúde da família $^{34}$, as quais podem identificar possíveis problemas no tratamento, como a adesão, bem como tendências suicidas dos mesmos.

Além disso, um fator a ser considerado no manejo de pacientes psiquiátricos são as consultas médicas. Segundo levantamento feito por Auchwski et al..$^{35}$, a maioria dos pacientes entrevistados não se lembrava das orientações recebidas pelos médicos, e também demonstravam não valorizá-las, ou então que estas mostraram-se insuficientes, o que pode favorecer recorrências e recaídas durante o tratamento da depressão e outros distúrbios psiquiátricos.
De acordo com a legislação relacionada aos medicamentos sujeitos a controle especial ${ }^{36}$, a quantidade de psicofármacos que pode ser adquirida com um receituário é para 60 dias de tratamento, de forma que o paciente pode ter acesso a uma grande quantidade dos mesmos. Portanto, pacientes psiquiátricos constituem um grupo de alto risco e uma das estratégias para prevenir qualquer ocorrências toxicológicas seria, além do apoio dos envolvidos, alterações na legislação, reduzindo o acesso a um número excessivo destes produtos.

Os analgésicos também representaram uma parcela bastante significativa nas intoxicações medicamentosas. Dentre estes, merece destaque o paracetamol por tratar-se de um fármaco de uso frequente e bem tolerado, mas, em casos de sobredose pode desencadear hepatotoxicidade com evolução fatal se o tratamento for negligenciado $^{37}$. Embora sejam raros os casos de exposição graves no Brasil, há um grande potencial de aumento dessas ocorrências, considerando o maior do consumo desse fármaco decorrente da epidemia nacional de dengue, demonstrando a importância de se realizar uma dispensação ativa deste fármaco, ainda que seja um medicamento isento de prescrição.

A exposição a mais de um medicamento, em geral, relaciona-se a uma maior intenção em cometer o suicídio. Nas tentativas de suicídio houve a presença de mais de uma substância em $38,0 \%$ e $42,2 \%$ dos casos em pesquisas realizadas por Bentur et al. ${ }^{38} \mathrm{e}$ Bernardes et al..$^{27}$, respectivamente. Muitas vezes as doses isoladas dos fármacos utilizados não são tóxicas, mas, a associação com outros medicamentos e/ou com outras substâncias químicas (como o álcool, por exemplo) pode ocasionar, por interação, aumento da toxicidade decorrente da potenciação dos efeitos, com agravamento do quadro clínico e necessidade de maior período de hospitalização ${ }^{27}$. O uso de múltiplos agentes não se deve apenas à impulsividade do paciente em recorrer aos que estão mais acessíveis, mas também à determinação em obter maior efeito tóxico e atingir doses letais, especialmente nos casos de ingestão de psicofármacos ${ }^{39,40}$.

Em relação às internações hospitalares, estudos demonstram que uma parcela significativa deve-se ao uso inadequado de medicamentos ${ }^{41}$. Nos Estados Unidos, durante o período de 1998 e 2006, Cox et al. ${ }^{42}$ observaram que as hospitalizações causadas por intoxicações envolvendo mulheres em idade fértil (faixa etária entre 14 a 44 anos) representaram 6,0\% do total de hospi- 
talizações ocorridas neste período. No presente estudo, houve necessidade de internação de 35,6\% das pacientes expostas a medicamentos, com uma média de permanência hospitalar de 2,5 dias. A maioria das hospitalizações foi decorrente de tentativas de suicídio e uso de psicofármacos, supondo-se nesses casos, que as pacientes foram acometidas por intoxicações mais graves e/ou que houve demora no atendimento após a ocorrência. A associação de dois ou mais fármacos relacionaram-se com maior tempo de internação, provavelmente, pelos efeitos sinérgicos entre as substâncias utilizadas.

Nesta pesquisa observou-se associação estatisticamente positiva entre a necessidade de internação de pacientes com nível superior. Talvez pacientes com maior escolaridade tenham mais acesso a medicamentos psicofármacos ou têm mais clareza de quais medicamentos geram maior risco de intoxicações, assim, nas exposições intencionais, estes apresentam condições mais graves, gerando a necessidade de internação.

A relação entre hospitalização e maior tempo entre a exposição e o atendimento reforça a necessidade de que os familiares ou os próprios pacientes, quando pertinente, devem ser orientados para que sob a mínima suspeita de intoxicação, os expostos devem ser imediatamente encaminhados aos serviços médicos de urgência e emergência. E estes preparados para prestar atendimento imediato aos pacientes em caso de suspeita de exposição a medicamentos, especialmente aos psicofármacos ou a mais de um medicamento, pois estes demonstraram gerar maior risco de internação.
A realização deste estudo fornece informações importantes para os profissionais de saúde, que poderão contribuir para aumentar a eficiência na triagem, intervenção e medidas de prevenção desses agravos na população feminina. A principal limitação desta pesquisa é inerente à utilização de dados retrospectivos e secundários, cujas informações em muitos campos das Fichas de Notificação apresentaram-se ignoradas.

As substâncias mais implicadas nas exposições analisadas foram os psicofármacos, os analgésicos e os anti-inflamatórios. Portanto, para reduzir as intoxicações agudas em mulheres nessa faixa etária suscetível, são necessários esforços que incluam orientação e conscientização dos profissionais prescritores e dos usuários sobre os possíveis riscos do uso indiscriminado desses fármacos, além de intervenções para reduzir os danos causados por lesões autoinfligidas.

Para uma maior compreensão dos fatores associados com intoxicações aguda em mulheres em idade fértil e em gestantes, e que possibilitem definir a população alvo e a elaboração de metas específicas para a intervenção, os estudos futuros devem incluir informações referentes à dose utilizada, para estabelecer a gravidade da intoxicação; à real intenção da ocorrência; ao tempo decorrido entre o conhecimento do estado gestacional e a exposição ao medicamento; história de transtorno mental ou abuso de substâncias psicoativas e, se a gravidez foi planejada ou não. Assim, esses estudos devem, além da análise dos sistemas de informação, realizar uma abordagem primária destes pacientes, familiares ou cuidadores, após as ocorrências.

\section{Colaboradores}

CH Takahama e CA Turini contribuíram na concepção do projeto, análise e interpretação dos dados e redação do artigo. E Girotto colaborou na análise e interpretação dos dados, revisão crítica e aprovação da versão final a ser publicada. 


\section{Referências}

1. Dall'Agnol RSA. Identificação e quantificação dos problemas relacionados com medicamentos em pacientes que buscam atendimento no serviço de emergência do HCPA [dissertação]. Porto Alegre: Universidade Federal do Rio Grande do Sul; 2004.

2. Marin N, Luiza VL, Osorio-de-Castro CGS, Machado-dos-Santos S. Assistência farmacêutica para gerentes municipais. 20a ed. Rio de Janeiro: OPAS, OMS; 2003.

3. Arrais PSD, Brito LL, Barreto ML, Coelho HLL. Prevalência e fatores determinantes do consumo de medicamentos no Município de Fortaleza, Ceará, Brasil. Cad Saude Publica 2005; 21(6):1737-1746.

4. Pereira JR, Soares L, Hoepfner L, Kruger KE, Guttervil ML, Tonini KC, Devegili DA, Rocha ER, Verdi F, Dalfovo D, Olsen K, Mendes T, Deretti R, Soares V, Lobermeyer C, Moreira J, Ferreira J, Francisco A. Riscos da automedicação: tratando o problema com conhecimento. In: Anais $3^{\circ}$ Seminário Integrado de Ensino, Pesquisa e Extensão - SIEPE; 2007; Joinville. [Internet]. [acessado 2012 Dez 28]. Disponível em: http://www.univille.edu.br

5. Vosgerau MZS, Soares DA, Souza RKT, Matsuo T, Carvalho GS. Consumo de medicamentos entre adultos na área de abrangência de uma Unidade Saúde da Família. Cien Saude Colet 2011; 16(Supl. 1):1629-1638.

6. Aquino DS. Por que o uso racional de medicamentos deve ser uma prioridade? Cien Saude Colet 2008; 13(Supl.):733-736.

7. Piercefield E, Archer P, Kemp P, Mallonee. Increase in Unintentional Medication Overdose Deaths Oklahoma, 1994-2006. Am J Prev Med 2010; 39(4):357363.

8. Mota DM, Melo JRR, Freitas DRC, Machado M. Perfil da mortalidade por intoxicação com medicamentos no Brasil, 1996-2005: retrato de uma década. Cien Saude Colet 2012; 17(1):61-70.

9. Organización Muldial de La Salud (OMS). Medicamentos: uso racional de lós medicamentos. Nota descriptiva No 338, Mayo de 2010. [Internet]. [acessado 2012 Nov 22]. Disponível em: http://www. who.int

10. Leite SN, Vieira M, Veber AP. Estudos de utilização de medicamentos: uma síntese de artigos publicados no Brasil e América Latina. Cien Saude Colet 2008; 13(Supl.):793-802.

11. Bochner R. Perfil das intoxicações em adolescentes no Brasil no período de 1999 a 2001. Cad Saude Publica 2006; 22(3):587-595.
12. Margonato FB, Thomson Z, Paoliello MMB. Determinantes nas intoxicações medicamentosas agudas na zona urbana de um município do sul do Brasil. Cad Saude Publica 2008; 24(2):333-341.

13. Margonato FB, Thomson Z, Paoliello MMB. Acute intentional and accidental poisoning with medications in a southern Brazilian city. Cad Saude Publica 2009; 25(4):849-856.

14. Bortoletto ME, Bochner R. Impacto dos Medicamentos nas Intoxicações Humanas no Brasil. Cad Saude Publica 1999; 15(4):859-869.

15. Bochner R, Souza VMFA. Panorama das intoxicações e envenenamentos registrados no Brasil pelo Sistema Nacional de Informações Tóxico-Farmacológicas (SINITOX). Rev Racine 2008; 106(5):44-58.

16. Brasil. Ministério da Saúde (MS). Fiocruz, Instituto de Comunicação e Informação Científica e Tecnológica em Saúde, Sistema Nacional de Informações Tóxico-Farmacológicas (SINITOX), Registro de intoxicações: dados nacionais. [Internet]. [acessado 05 dez 2012]. Disponível em: http://www. fiocruz.br

17. Brasil. Ministério da Saúde (MS). Secretaria de Vigilância em Saúde, Sistema de Informação de Agravos de Notificação - Sinan Net, Tabulação de dados sobre Intoxicações exógenas. [Internet]. [acessado 2012 dez 10]. Disponível em: http://datasus. gov.br

18. Schillie SF, Shehab N, Thomas KE, Budnitz DS. Medication Overdoses Leading to Emergency Department Visits Among Children. Am J Prev Med 2009; 37(3):181-187.

19. Chen F, Wen J, Wang X, Lin Q, Lin C. Epidemiology and characteristics of acute poisoning treated at an emergency Center. World J Emerg Med 2010; 1(2):154-156.

20. Warner M, Chen LH, Makuc DM, Anderson RN, Miniño AM. Drug Poisoning Deaths in the United States, 1980-2008. U.S. Department of Health \& Human Services. Hyattsville: Centers for Disease Control and Prevention. National Center for Health Statistics. NCHS Data Brief 2011; 81:1-8.

21. Xiang Y; Zhao W; Xiang H.; Smith GA. ED visits for drug-related poisoning in the United States, 2007. Am J Em Med 2012; 30(2):293-301. 
22. Chrisman JR, Silva JJO, Martins EV, Bochner R, Ferreira RGSS, Ferrante ACP, Alves SR. Avaliação da sub-notificação dos casos de intoxicação humana através de diferentes sistemas de informação e bases de dados. In: IV Bienal de Pesquisa da FIOCRUZ; 2004; Rio de Janeiro. [Internet]. [acessado 2013 Jan 09]. Disponível em: http://www.fiocruz.br

23. Brasil. Ministério da saúde (MS). Secretaria de Atenção à Saúde, Departamento de Ações Programáticas Estratégicas. Política Nacional de Atenção Integral à Saúde da Mulher: Princípios e Diretrizes. $1^{\text {a }}$ ed. Brasília: Ministério da Saúde; 2011. 82 p. [Internet]. [acessado 2013 jan 20]. Disponível em: http:/ /saude.gov.br

24. World Health Organization (WHO). WHO Collaborating Centre for Drug Statistics Methodology. ATC/DDD index 2012. [Internet]. [acessado 2013 jun 01]. Disponível em: http://www.who.int

25. Iribarren C, Sidney S, Jacobs Júnior DR, Weisner C. Hospitalization for suicide attempt and completed suicide: epidemiological features in a managed care population. Soc Psychiatry Psychiatr Epidemiol 2000; 35(7):288-296.

26. Fernandes GF Palvo, FA Pinton, Dourado DAN, Mendes CAC. Impacto das intoxicações por antidepressivos tricíclicos comparados aos depressores do "sistema nervoso central". Arq Ciênc Saúde 2006; 13(3):61-65.

27. Bernardes SS, Turini CA, Matsuo T. Perfil das tentativas de suicídio por sobredose intencional de medicamentos atendidas por um Centro de Controle de Intoxicações do Paraná, Brasil. Cad Saude Publica 2010; 26(7):1366-1372.

28. McClure CK, Katz KD, Patrick TE, Kelsey, SF, Weiss, HB. The Epidemiology of Acute Poisonings in Women of Reproductive Age and During Pregnancy, California, 2000 - 2004. Matern Child Health J 2011; 15(7):964-973.

29. Melione LPR, Mello-Jorge MHP. Gastos do Sistema único de saúde com Internações por Causas Externas em São José dos Campos, São Paulo, Brasil. Cad Saude Publica 2008; 24(8):1814-1824.

30. Marcondes Filho W, Mezzaroba L, Turini CA, Koike A, Motomatsu JRA, Shibayama EM, Fenner F. Tentativas de suicídio por substâncias químicas na adolescência e juventude. Adolesc Latinoam 2002; 3(2).

31. Avanci RC, Pedrão LJ, Costa Júnio ML. Perfil do adolescente que tenta suicídio em uma unidade de emergência. Rev Bras Enferm 2005; 58(5):535-539.
32. Rodrigues MAP, Facchini LA, Lima MS. Modificações nos padrões de consumo de psicofármacos em localidade do Sul do Brasil. Rev Saude Publica 2006; 40(1):107-114.

33. Bertolote JM, Mello-Santos C, Botega NJ. Detecção do risco de suicídio nos serviços de emergência psiquiátrica. Rev Bras Psiquiat 2010; 32(Supl. 2):87-95.

34. Pajonk FG, Guenberg KA, Moecke H, Naber D. Suicides and suicide attempts in emergency medicine. Crisis 2002; 23(2):68-73.

35. Auchwski L, Andreatini R, Galduróz JCF, Lacerda RB. Avaliação da orientação médica sobre os efeitos colaterais de benzodiazepínicos. Rev Bras Psiquiat 2004 26(1):24-31.

36. Brasil. Ministério da Saúde (MS). Agência Nacional de Vigilância Sanitária. Portaria n. ${ }^{\circ} 344$, de 12 de maio de 1998. Aprova o Regulamento Técnico sobre substâncias e medicamentos sujeitos a controle especial. Diário Oficial da União 1998; 12 maio.

37. Schilling A, Corey R, Leonard M, Eghtesad B. Acetaminophen: old drug, new warnings. Clev Clinic J Med 2010; 77(1):19-27.

38. Bentur Y, Raikhlin-Eisenkraft B, Lavee M. Toxicological features of deliberate self-poisonings. Hum Exp Toxicol 2004; 23(7):331-337.

39. Carson HJ. Classes of drugs and their prevalence in multiple drug intoxication in suicides and accidents. Leg Med 2008; 10(2):92-95.

40. Veldhuizen S, Terrance W, Cairney J. Alcohol consumption among Canadians taking benzodiazepines and relate drugs. Pharmacoepidem Drug Safe 2009; 18(3):203-210.

41. Mastroianni PC, Varallo FR, Barg MS, Noto AR, Galduróz JCF. Contribuição do uso de medicamentos para a admissão hospitalar. Braz J Pharm Sci 2009; 45(1):163-170.

42. Cox S, C Kuo, DJ Jamieson, AP Kourtis, McPheeters ML, Meikle SF, Posner SF. Poisoning hospitalisations among reproductive-aged women in the USA, 1998-2006. Inj Prev 2011; 17(5):332-337.

Artigo apresentado em 16/03/2013

Aprovado em 27/04/2013

Versão final apresentada em 11/05/2013 
